\title{
Oncological outcomes of high-grade T1 non-muscle-invasive bladder cancer treatment in octogenarians
}

\author{
Aleksander Ślusarczyk ${ }^{1} \cdot$ Karolina Garbas $^{1}$ (1) $\cdot$ Piotr Zapała $^{1} \cdot$ Łukasz Zapała $^{1} \cdot$ Piotr Radziszewski $^{1}$
}

Received: 29 January 2021 / Accepted: 20 April 2021 / Published online: 26 April 2021

(c) The Author(s) 2021

\begin{abstract}
Purpose To evaluate the outcomes of high-grade T1 non-muscle-invasive bladder cancer treatment (NMIBC) in elderly patients over 80 years of age.

Methods This is a retrospective single tertiary-centre study. Medical records of patients with T1 high-grade NMIBC treated with transurethral resection of the bladder tumour (TURBT) were reviewed. Among 269 patients with high-grade T1 NMIBC, 74 individuals were over 80 years of age at the time of surgery. Finally, 67 patients met the inclusion criteria.

Results Only $47.8 \%$ of patients $(N=32)$ received at least five of the six instillations of the BCG immunotherapy induction course. Oncological outcomes were compared between patients who received at least the induction course of BCG and non-BCG-treated patients matched to each other based on age and Charlson comorbidity index. Thirty case-control pairs were included in the final analysis. Rates of disease recurrence ( $80 \%$ vs. $53 \%)$ and cancer-specific mortality (40\% vs. $10 \%)$ were significantly higher in the group of patients who did not receive BCG. BCG therapy, Charlson comorbidity index, haemoglobin concentration and the number of tumours $>3$ in TURBT constituted independent prognostic factors for cancerspecific survival (CSS).
\end{abstract}

Conclusion BCG should be strongly recommended to patients with T1HG NMIBC despite advanced age and comorbidities. Already BCG induction improves CSS and reduces the recurrence rate in octogenarians with T1HG bladder cancer.

Keywords Non-muscle-invasive bladder cancer $\cdot$ BCG therapy $\cdot$ Elderly patients $\cdot$ Survival $\cdot$ Advanced age

\section{Introduction}

Bladder cancer is the eleventh most common malignancy worldwide [1]. In general, most patients present with nonmuscle-invasive bladder cancer (NMIBC) regarded as tumour confined to the mucosa (Ta or carcinoma in situ) or the submucous layer of the urinary bladder wall (T1), which might be effectively managed with transurethral resection of the bladder tumour (TURBT) [1]. However, in NMIBC characterized as high-risk of progression (high-grade tumour, T1 category tumour or CIS), adjuvant treatment with intravesical Bacillus Calmette-Guerin (BCG) vaccine instillations is necessary to improve recurrence- (RFS) and progressionfree survival (PFS) [2, 3]. High-grade T1 bladder tumour

Karolina Garbas

kgarbas98@gmail.com

1 Department of General, Oncological and Functional Urology, Medical University of Warsaw, Lindleya 4, 02-005 Warsaw, Poland constitutes the most aggressive form of NMIBC with the highest risk for recurrence and progression $[4,5]$.

Patients' age represents one of the greatest single risk factors for bladder cancer (BC) development [6]. The prevalence of bladder cancer is highest in an elderly population, with 73 years being an average age of diagnosis and peak incidence reported at 85 years old [7,8]. The burden of $\mathrm{BC}$ in elderlies might be expected to grow as further ageing of the population is being observed [6]. BC patients over 80 years of age often present with several comorbidities, with cardiovascular diseases being the most frequent. Elderly patients require more individualized strategies to prevent disease progression without causing serious treatment complications and compromising their quality of life. Due to advanced age, comorbidities and disability, these patients are not always fitting or eager to obtain optimal recommended oncological treatment.

Relatively little is known regarding the efficacy of NMIBC treatment in elderly patients [9]. Several studies report a measurable impact of ageing on the efficacy of 
intravesical BCG therapy [10-12]. Results of recent reports suggest that the BCG therapy for NMIBC is less effective in patients $>70$ years of age compared to younger ones [11]. More importantly, the study included only 20 patients aged 80 years and more, which was previously depicted as a threshold of particular BCG failure risk [10].

The potential complications of intravesical therapy might not be as well-tolerated in elderly individuals whose urinary, cardiovascular and immunological functions, as well as a pulmonary reserve, are decreased [6]. Some investigators caution against intravesical therapy in the elderly and suggest that due to complications, BCG maintenance should be avoided in the population $>80$ years $[6,13]$.

Available studies encompassing a larger cohort of elderly NMIBC patients are lacking and guidelines do not recommend any treatment modification in that group. To date, subcohorts of large clinical trials provide limited insight into outcomes of BCG administration in patients older than 80 years [11].

In this study, we aimed to evaluate the results of T1HG bladder cancer treatment in patients over 80 years of age and assess the impact of intravesical BCG therapy on oncological outcomes.

\section{Methods}

This is a single tertiary-centre case-control study. Medical records of patients who underwent transurethral resection of the bladder tumour in our department between 2010 and 2018 were retrospectively reviewed. Patients with T1 highgrade tumour in TURBT specimen and at least 80 years of age at the time of surgery were identified.

The surgeries were carried out according to the protocol recommended by the European Association of Urology (EAU) clinical guideline [1]. Surgical specimens were reviewed by a genitourinary pathologist, graded according to the 1973 and 2004 WHO grading system and staged according to the 2009 TNM classification.

Consecutive patients were treated with adjuvant BCG immunotherapy in accordance with EAU guidelines [1]. BCG induction included 6 weekly instillations, whereas the maintenance course consisted of 3 weekly instillations administered at 3, 6, 12, 18, 24, 30 and 36 months. Ninetyseven percent of patients $(N=31)$ were treated with RIVM BCG strain, with each dose including at least $2 \times 10^{8}$ and no more than $3 \times 10^{9}$ BCG. One patient (3\%) was managed with Moreau strain, with each dose containing at least $3 \times 10^{8}$ BCG. Patients always received a full dose of BCG.

Baseline characteristics including clinical, histopathological and laboratory data were collected. Histopathological data included staging and grading of each specimen. Clinical data included age, gender, previous history of bladder cancer, tumour size, multifocality and comorbidities. Each patient was retrospectively classified using Charlson comorbidity index (CCI) [14] and NMIBC dedicated prognostic tools (European Organization for Research and Treatment of Cancer (EORTC) risk tables and Spanish Urological Club for Oncological Treatment (CUETO) scoring model). Laboratory data included preoperative blood parameters - blood cell counts and haemoglobin concentration collected from pretreatment laboratory test.

Differences between groups were evaluated with the $U$ Mann-Whitney test for continuous variables and Fischer's exact test or chi-square test for categorical variables. Continuous variables are presented as median values accompanied by the interquartile range (IQR). For prediction analysis, univariable and multivariable Cox proportional hazard was used. The differences in time to event were evaluated with log-rank test and illustrated with Kaplan-Meier curves. Correlations between variables were checked using the Spearman test. For all statistical analyses, a two-sided $p$ value $<0.05$ was considered statistically significant. Statistical analysis was performed with the SAS System (version 9.4).

\section{Results}

Among 269 patients with high-grade T1 non-muscle-invasive bladder cancer, 74 individuals were over 80 years of age at the time of surgery. Patients with T2HG at restaging TURBT (re-TURBT) $(N=4)$, patients without complete macroscopic resection $(N=2)$ and lack of follow-up $(N=1)$ were excluded from the analysis. Finally, sixty-seven patients met the inclusion criteria.

During a median of 29-month follow-up, 44 patients recurred and 26 patients developed progression. Only $47.8 \%$ of patients $(N=32)$ received at least five of six instillations of BCG immunotherapy induction course, whereas $29.9 \%$ of patients $(N=20)$ were treated with at least one maintenance BCG course. In the remaining $52.2 \%$ of patients $(N=35)$ who did not receive BCG, the most common causes were patient's non-compliance (34.3\%), presence of comorbidities including uncontrolled systemic diseases (17.1\%), and guidelines non-adherence due to suspected non-compliance (14.3\%). Other less frequent causes were BCG intolerance (5.7\%), dementia (5.7\%), mobility impairment (5.7\%), delay in BCG initiation (2.9\%) and unknown reason (8.6\%).

Oncological outcomes were compared between patients who received at least the induction course of BCG and nonBCG-treated patients matched to each other based on age and Charlson comorbidity index. Patients selected after case-control matching included 30 BCG-treated and 30 nontreated individuals, as presented in Table 1. Further analysis 
Table 1 Descriptive characteristics of patients with T1HG bladder cancer over 80 years at the time of surgery

\begin{tabular}{|c|c|c|c|c|c|c|c|}
\hline & \multicolumn{2}{|l|}{ Overall } & \multicolumn{2}{|l|}{ Non-BCG group } & \multicolumn{2}{|c|}{ BCG group } & \multirow[t]{2}{*}{$p$ value } \\
\hline & $\begin{array}{l}\text { No. Pts./ } \\
\text { Median }\end{array}$ & $\% / \mathrm{IQR}$ & No. Pts./Median & $\% / \mathrm{IQR}$ & $\begin{array}{l}\text { No. Pts./ } \\
\text { Median }\end{array}$ & $\% / \mathrm{IQR}$ & \\
\hline \multicolumn{8}{|l|}{ Characteristics } \\
\hline Male gender & 50 & 83.3 & 22 & 73.3 & 28 & 86.7 & NS \\
\hline Grade 3 & 50 & 83.3 & 22 & 73.3 & 28 & 93.3 & 0.08 \\
\hline Concomitant CIS & 7 & 11.7 & 1 & 3.3 & 6 & 20 & 0.047 \\
\hline Recurrent tumour & 24 & 40 & 11 & 36.7 & 13 & 43.3 & NS \\
\hline Multiple tumours & 35 & 58.3 & 20 & 66.7 & 15 & 50 & NS \\
\hline More than three tumours & 19 & 31.7 & 9 & 30 & 10 & 33.3 & NS \\
\hline Big tumour $\geq 3 \mathrm{~cm}$ & 27 & 45 & 16 & 53.3 & 11 & 36.7 & NS \\
\hline No muscle in TURBT specimen & 9 & 15 & 5 & 16.7 & 4 & 13.3 & NS \\
\hline HG tumour history & 15 & 25 & 9 & 30 & 6 & 20 & NS \\
\hline ReTURBT performance & 43 & 71.7 & 20 & 66.7 & 23 & 76.7 & NS \\
\hline Tumour in reTURBT & 22 & 36.7 & 8 & 40 & 14 & 60.9 & 0.18 \\
\hline HG tumour in reTURBT & 25 & 41.7 & 8 & 25 & 17 & 56.5 & $\mathbf{0 . 0 3 7}$ \\
\hline \multicolumn{8}{|l|}{ Comorbidities } \\
\hline Hypertension & 49 & 81.7 & 26 & 86.7 & 23 & 76.7 & NS \\
\hline Coronary artery disease & 39 & 65 & 15 & 50 & 24 & 80 & 0.014 \\
\hline Myocardial infarction & 12 & 20 & 4 & 13.3 & 8 & 26.7 & NS \\
\hline COPD & 6 & 10 & 3 & 10 & 3 & 10 & NS \\
\hline Diabetes mellitus type 2 & 17 & 28.3 & 10 & 33.3 & 7 & 23.3 & NS \\
\hline Dementia & 2 & 3.3 & 2 & 6.7 & 0 & 0 & 0.15 \\
\hline History of other cancer & 11 & 18.3 & 2 & 6.7 & 9 & 30 & 0.020 \\
\hline Stroke & 17 & 28.3 & 9 & 30.5 & 8 & 25.4 & NS \\
\hline Atrial fibrillation & 14 & 23.3 & 6 & 20 & 8 & 26.7 & NS \\
\hline Heart failure & 17 & 28.3 & 8 & 26.7 & 9 & 30 & NS \\
\hline UTUC & 3 & 5 & 2 & 6.7 & 1 & 3.3 & NS \\
\hline \multicolumn{8}{|l|}{ Other } \\
\hline Age & 85 & $82-89$ & 85 & $81-89.5$ & 85 & $82.5-88$ & NS \\
\hline Charlson comorbidity index & 7 & $6-8$ & 7 & $6-8$ & 7 & $6-8$ & NS \\
\hline ASA & 3 & $3-3$ & 3 & $3-3$ & 3 & $3-3$ & NS \\
\hline Haemoglobin (g/L) & 128 & $116-141.5$ & 129.5 & $113-143$ & 127 & 119-139 & NS \\
\hline CUETO recurrence score & 8 & $5-10$ & 7.5 & $5-10$ & 8 & $5-11$ & NS \\
\hline CUETO progression score & 9 & $8-10$ & 8 & $8-10$ & 9 & $8-10$ & 0.09 \\
\hline EORTC recurrence score & 8 & $5-9$ & 8 & $6-9$ & 7 & $5-8$ & NS \\
\hline EORTC progression score & 14 & $9.5-15$ & 14 & $9-15$ & 14 & $11-15$ & NS \\
\hline \multicolumn{8}{|l|}{ Outcomes } \\
\hline Recurrence & 40 & 66.7 & 24 & 80 & 16 & 53.3 & 0.029 \\
\hline Progression & 24 & 40 & 15 & 50 & 9 & 30 & 0.11 \\
\hline All-cause death & 33 & 55 & 20 & 66.7 & 13 & 43.3 & 0.07 \\
\hline Cancer-specific death & 15 & 25 & 12 & 40 & 3 & 10 & 0.007 \\
\hline
\end{tabular}

Comparison between BCG-treated $(N=30)$ and non-treated groups $(N=30)$

$C I S$ carcinoma in situ, $H G$ high-grade, reTURBT restaging transurethral resection of bladder tumour, COPD chronic obstructive pulmonary disease, ASA American Society of Anesthesiologists Score, IQR interquartile range, The bold values denote statistical significance at the $\mathrm{p}<0.05$ level

was performed on a cohort of 60 case-control matched patients, in whom 40 recurrences and 24 progressions were observed.
The presence of concomitant CIS $(p=0.047)$, residual high-grade tumour in re-TURBT $(p=0.037)$, history of other cancer $(p=0.020)$ and coronary artery disease 
$(p=0.014)$ were more frequent in the BCG-treated cohort. Both groups did not differ significantly in terms of other major factors that could influence oncological outcomes and overall survival.

Rates of disease recurrence ( $80 \%$ vs 53\%) and cancerspecific mortality ( $40 \%$ vs $10 \%$ ) were significantly higher in the group of patients who did not receive BCG compared to BCG-treated ones.

In Kaplan-Meier analysis, BCG induction was associated with favourable cancer-specific survival (CSS) (Fig. 1). We did not observe the association between BCG induction course and the time to recurrence or progression, though (Fig. 1). However, the higher number of received BCG courses was associated with better RFS in multivariate analysis. EORTC or CUETO risk scores did not predict the RFS or PFS in our study population. Univariate analysis for CSS was performed (see Table 2 for details). Cox proportional hazards were utilized to investigate which factors are independently associated with RFS, PFS, CSS and overall survival (OS) (see Table 3 for details). There were no correlations between the variables included in each model.
Clinically significant side effects of BCG administration were observed in $32 \%$ of patients $(N=11)$. This included sudden cardiac arrest $(N=1)$, systemic side effects such as inflammatory response $(N=2)$ and local side effects $(N=8)$. BCG intolerance, regarded as withdrawal due to severe side effects, occurred in six patients.

\section{Discussion}

In our study, we analysed the oncological outcomes of T1HG NMIBC in patients older than 80 years at the time of TURBT. We aimed to identify factors associated with compromised cancer-specific survival in the geriatric population of T1HG patients. Cases were matched to controls based on age and comorbidities, leading to the inclusion of 30 BCGtreated and 30 non-treated patients into the final analysis.

Intravesical BCG therapy, low Charlson comorbidity index, higher haemoglobin concentration and three or fewer tumours in TURBT were associated with better cancer-specific survival. Haemoglobin was previously reported as OS

$\mathrm{P}=0.03$
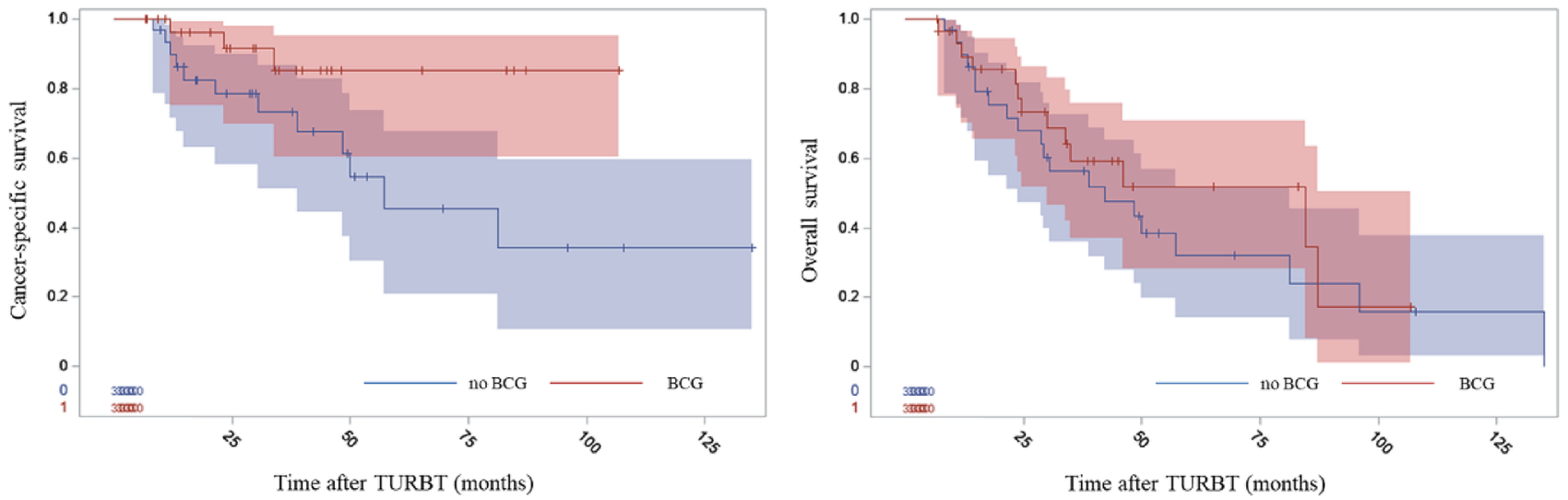

$\mathrm{P}=0.32$
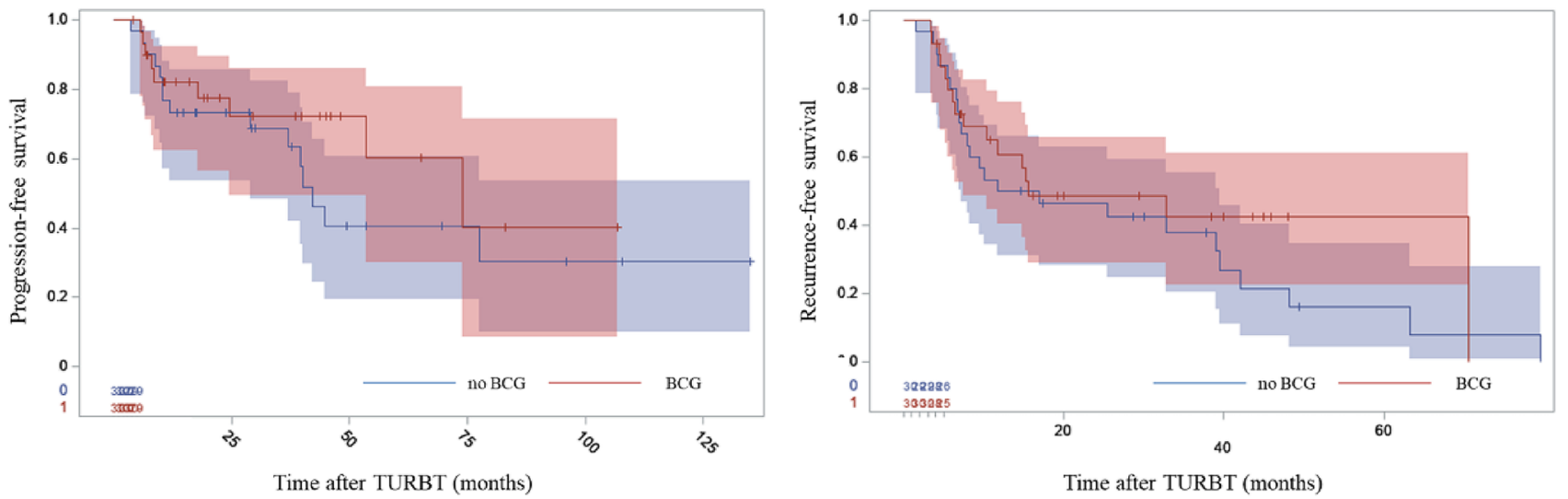

Fig. 1 Kaplan-Meier curves representing the influence of induction BCG therapy on oncological outcomes and overall survival 
Table 2 Univariate Cox proportional hazard regression analysis for prediction of cancer-specific survival in T1HG patients $>80$ years

\begin{tabular}{|c|c|c|c|c|}
\hline Clinicopathological features & & HR & $95 \% \mathrm{CI}$ & $p$ \\
\hline Age & & 1.046 & $0.92-1.19$ & NS \\
\hline BCG induction & (Yes vs no) & 0.271 & $0.08-0.96$ & 0.044 \\
\hline Number of BCG courses & & 0.514 & $0.26-1.04$ & 0.062 \\
\hline Male gender & (Yes vs no) & 0.350 & $0.12-1.03$ & 0.057 \\
\hline Grade (yes vs no) & (3 vs 2$)$ & 4.389 & $0.56-34.08$ & 0.16 \\
\hline Concomitant CIS & (Yes vs no) & 0.602 & $0.08-4.73$ & NS \\
\hline Recurrent tumour & (Yes vs no) & 0.994 & $0.35-2.85$ & NS \\
\hline Multiple tumours & (Yes vs no) & 1.724 & $0.59-5.08$ & NS \\
\hline Tumour size $\geq 3 \mathrm{~cm}$ & (Yes vs no) & 2.240 & $0.79-6.34$ & 0.13 \\
\hline $\begin{array}{l}\text { Muscle in TURBT speci- } \\
\text { men }\end{array}$ & (No vs yes) & 2.998 & $0.95-9.48$ & 0.062 \\
\hline No. of tumours & $(>3$ vs $\leq 3)$ & 2.409 & $0.81-7.14$ & 0.11 \\
\hline HG tumour history & (Yes vs no) & 1.381 & $0.47-4.07$ & NS \\
\hline reTURBT & (Yes vs no) & 0.635 & $0.22-1.87$ & NS \\
\hline Tumour in reTURBT & (Yes vs no) & 1.757 & $0.48-6.48$ & NS \\
\hline HG tumour in reTURBT & (Yes vs no) & 2.031 & $0.57-7.23$ & NS \\
\hline \multicolumn{5}{|l|}{ Comorbidities } \\
\hline Hypertension & (Yes vs no) & 5.445 & $0.70-42.11$ & 0.10 \\
\hline Coronary artery disease & (Yes vs no) & 0.661 & $0.24-1.84$ & NS \\
\hline Myocardial infarction & (Yes vs no) & 1.188 & $0.33-4.25$ & NS \\
\hline COPD & (Yes vs no) & 1.765 & $0.60-5.18$ & NS \\
\hline Diabetes mellitus type 2 & (Yes vs no) & 0.833 & $0.27-2.62$ & NS \\
\hline History of other cancer & (Yes vs no) & 1.020 & $0.29-3.63$ & NS \\
\hline Atrial fibrillation & (Yes vs no) & 0.631 & $0.18-2.24$ & NS \\
\hline Heart failure & (Yes vs no) & 1.765 & $0.60-5.12$ & NS \\
\hline UTUC & (Yes vs no) & 5.445 & $0.70-42.11$ & 0.10 \\
\hline \multicolumn{5}{|l|}{ Other } \\
\hline $\begin{array}{l}\text { Charlson comorbidity } \\
\text { index }\end{array}$ & & 1.514 & $0.98-2.35$ & 0.064 \\
\hline ASA & & 1.104 & $0.56-2.18$ & NS \\
\hline Haemoglobin & & 0.976 & $0.95-1.00$ & 0.089 \\
\hline NLR & & 1.080 & $0.88-1.33$ & NS \\
\hline CUETO recurrence score & & 1.119 & $0.95-1.31$ & 0.17 \\
\hline CUETO progression score & & 1.186 & $0.86-1.63$ & NS \\
\hline EORTC recurrence score & & 1.207 & $1.00-1.46$ & 0.051 \\
\hline EORTC progression score & & 1.095 & $0.96-1.25$ & NS \\
\hline
\end{tabular}

$H R$ hazard ratio, CI confidence interval, CIS carcinoma in situ, reTURBT restaging transurethral resection of bladder tumour, $C O P D$ chronic obstructive pulmonary disease, UTUC upper tract urothelial cancer, ASA American Society of Anaesthesiologists Score, NLR neutrophil-to-lymphocyte ratio, The bold values indicate $\mathrm{p}<0.1$ values

and CSS predictor in the cohort of NMIBC patients [15, 16], and our study validates its prognostic role in elderly T1HG individuals. Our observations underline the pivotal role of BCG treatment and provide evidence that BCG administration is associated with prolonged CSS in the group of elderly $>80$ years. Suboptimal BCG use in elderly patients remains a significant problem observed not only in our study
Table 3 Multivariate Cox proportional hazard regression analysis for prediction of (1) cancer-specific survival, (2) overall survival, (3) progression-free survival and (4) recurrence-free survival in T1HG patients $>80$ years

\begin{tabular}{llll}
\hline & \multicolumn{3}{l}{ Multivariate analysis } \\
\cline { 2 - 4 } & HR & $95 \%$ CI & $p$ \\
\hline CSS & & & \\
BCG induction therapy & 0.234 & $0.06-0.87$ & 0.030 \\
No BCG therapy & Reference & & \\
Charlson comorbidity index & 1.711 & $1.04-2.82$ & 0.035 \\
Haemoglobin & 0.962 & $0.96-0.93$ & 0.014 \\
No. of tumours $>3$ & 3.173 & $1.00-10.09$ & 0.050 \\
No. of tumours $\leq 3$ & Reference & & \\
OS & & & \\
Multiple tumours & 2.979 & $1.32-6.75$ & 0.009 \\
Single tumour & Reference & & \\
Charlson comorbidity index & 1.380 & $0.97-1.96$ & 0.074 \\
Haemoglobin & 0.964 & $0.94-0.98$ & 0.0004 \\
PFS & & & \\
Tumour size $\geq 3$ cm & 2.387 & $1.03-5.53$ & 0.042 \\
Tumour size $<3$ cm & Reference & & \\
Charlson comorbidity index & 1.579 & $1.09-2.28$ & 0.015 \\
RFS & & & \\
BCG-number of courses & 0.776 & $0.61-0.99$ & 0.038 \\
$\quad$ reTURBT & & & \\
$\quad$ Not performed & 3.720 & $1.52-9.09$ & 0.004 \\
$\quad$ Residual tumour & 2.829 & $6.54-1.23$ & 0.015 \\
$\quad$ No residual tumour & Reference & & \\
\hline
\end{tabular}

$H R$ hazard ratio, $C I$ confidence interval, reTURBT restaging transurethral resection of bladder tumour

$[17,18]$. It has been already reported that in the large cohort of 23,932 eligible NMIBC patients above 65 years, only $22 \%$ received adjuvant BCG [17]. In the same study, only $39 \%$ of patients with stage CIS or T1 received BCG. In a Swedish cohort of patients with $\mathrm{T} 1$ pathology, only $24 \%$ were treated with BCG [19]. Moreover, another study demonstrated that among 1590 patients who underwent a single resection of high-grade tumour 59.6\% did not receive any BCG therapy, whereas $25.8 \%$ received only partial induction. Multiple resections and younger chronological age, but not the presence of comorbidities, were associated with BCG initiation [18]. In several studies, age $>80$ years was found to increase the risk of not receiving BCG $[17,18]$. Unexpectedly, in our study encompassing only patients older than 80 years, neither age nor the presence of comorbidities were related to BCG administration. Residual high-grade tumour at restaging TURBT and concomitant carcinoma in situ were associated with receiving at least BCG induction course.

BCG immunotherapy remains a gold standard therapy, which prevents tumour recurrence and progression [2, 3]. Although some evidence suggested a lower efficacy 
of BCG in an older subgroup of patients [10-12], guidelines strongly recommend BCG initiation regardless of age and comorbidities [1]. Till date, the survival benefit from BCG therapy is not as well evidenced as its efficacy in preventing recurrence and progression [2, 3, 20]. Our analysis confirms the CSS benefit from receiving at least BCG induction, which underlies the importance of BCG initiation in high-risk patients regardless of age. Moreover, in our study, patients treated with BCG compared to the control group presented more frequently with concomitant CIS and had higher rates of residual high-grade tumour in re-TURBT and still achieved better CSS.

The recurrence rate was significantly lower $(80 \%$ vs $53 \% ; p=0.03)$ and the rate of progression $(50 \%$ vs $30 \%$; $p=0.11$ ) seemed to be lower in the BCG-treated group, as the relationship tended to statistical significance. Residual tumour at re-TURBT, lack of re-TURBT performance and number of received BCG courses constituted independent predictors of RFS, whereas tumour size $\geq 3 \mathrm{~cm}$ and presence of comorbidities (assessed using the Charlson comorbidity index) were associated with PFS. This emphasizes the fundamental role of BCG administration in preventing disease recurrence and indicates that big tumours $(\geq 3 \mathrm{~cm})$ are characterized by the increased potential to infiltrate the muscle layer of the urinary bladder wall. Big tumour size was already shown as an independent prognostic factor for PFS in the T1HG BC population [21]. Inclusion of reTURBT performance as an independent predictor of RFS underlies its substantial role in high-risk NMIBC treatment, even though it was not associated with CSS benefit. Re-TURBT is therefore a crucial procedure for therapeutic and staging purposes [22, 23].

Notably, $79 \%$ of patients who progressed to muscleinvasive disease and were dead at the last follow-up died from bladder cancer. Such observation might be attributed to the frequent presence of cardiovascular diseases and other comorbidities in the studied population, which were the actual causes of death in the remaining $21 \%$ of patients. Independent factors associated with overall survival were the following: Charlson comorbidity index, haemoglobin concentration and the presence of multiple tumours. In the Kaplan-Meier analysis, we did not observe overall survival benefit related to BCG use, but this might result from a small sample size. On the other hand, a small but significant survival advantage associated with BCG in the elderlies was reported by Spencer et al. [17].

As there was no correlation between the Charlson comorbidity index and BCG administration, CCI appeared to be an independent prognostic factor for oncological outcomes (PFS and CSS) and, unsurprisingly, for overall survival. Therefore, routine CCI estimation might be of clinical use in geriatric bladder cancer patients to guide suitable individualized therapy and follow-up. It has been already reported that
CCI might be utilized as a predictor of radical cystectomy outcomes [24]. The impact of comorbidities on treatment effectiveness and compromised compliance among cancer patients has already been summarized by Søgaard et al. [25].

In the present study, we observed suboptimal BCG use with less than half of patients receiving at least induction BCG therapy. Efforts should be made to increase the rate of BCG receivers among elderlies with high-risk NMIBC and improve patient's compliance without the quality of life impairment, which might be even a greater challenge in the era of Covid-19 pandemic.

\section{Conclusion}

In our study, we confirm the utmost importance of BCG therapy initiation in elderly high-risk NMIBC patients. To conclude, BCG should be strongly recommended to patients with T1HG despite advanced age and comorbidities. Already BCG induction improves cancer-specific survival and reduces the recurrence rate in T1HG patients over 80 years of age.

\section{Study limitations}

Limitations of the study result from its retrospective design. The follow-up was inconsistent among patients and the sample size was small. Lack of significant association between RFS, PFS and BCG use might be attributed to the cohort size and relatively short, inconsistent follow-up in many cases. Censored data at the time of the last follow-up and a wide $95 \%$ confidence interval might also contribute to the lack of statistically significant PFS differences.

\section{Declarations}

Conflict of interest The authors declare that they have no conflict of interest.

Ethical approval All procedures performed during the study were in accordance with the ethical standards of the institutional and national research committee and with the 1964 Helsinki Declaration and its later amendments.

Open Access This article is licensed under a Creative Commons Attribution 4.0 International License, which permits use, sharing, adaptation, distribution and reproduction in any medium or format, as long as you give appropriate credit to the original author(s) and the source, provide a link to the Creative Commons licence, and indicate if changes were made. The images or other third party material in this article are included in the article's Creative Commons licence, unless indicated otherwise in a credit line to the material. If material is not included in 
the article's Creative Commons licence and your intended use is not permitted by statutory regulation or exceeds the permitted use, you will need to obtain permission directly from the copyright holder. To view a copy of this licence, visit http://creativecommons.org/licenses/by/4.0/.

\section{References}

1. Professionals S-O. EAU guidelines: non-muscle-invasive bladder cancer [Internet]. Uroweb. https://uroweb.org/guideline/non-muscle-invasive-bladder-cancer/. Accessed 28 Dec 2020

2. Böhle A, Jocham D, Bock PR (2003) Intravesical bacillus Calmette-Guerin versus mitomycin $\mathrm{C}$ for superficial bladder cancer: a formal meta-analysis of comparative studies on recurrence and toxicity. J Urol 169(1):90-95

3. Böhle A, Bock PR (2004) IntravesicalbacilleCalmette-Guérin versus mitomycin $\mathrm{C}$ in superficial bladder cancer: formal metaanalysis of comparative studies on tumor progression. Urology 63(4):682-686 (discussion 686-687)

4. Ślusarczyk A, Zapała P, Zapała $Ł$, Piecha T, Radziszewski P (2019) Prediction of BCG responses in non-muscle-invasive bladder cancer in the era of novel immunotherapeutics. IntUrolNephrol 51(7):1089-1099

5. Cambier S, Sylvester RJ, Collette L, Gontero P, Brausi MA, van Andel $\mathrm{G}$ et al (2016) EORTC nomograms and risk groups for predicting recurrence, progression, and disease-specific and overall survival in non-muscle-invasive stage Ta-T1 urothelial bladder cancer patients treated with 1-3 years of maintenance Bacillus Calmette-Guérin. EurUrol 69(1):60-69

6. Shariat SF, Sfakianos JP, Droller MJ, Karakiewicz PI, Meryn S, Bochner BH (2010) The effect of age and gender on bladder cancer: a critical review of the literature. BJU Int 105(3):300-308

7. Schultzel M, Saltzstein SL, Downs TM, Shimasaki S, Sanders C, Sadler GR (2008) Late age ( $\geq 85$ years) peak incidence of urinary bladder cancer. J Urol 179(4):1302-1306

8. Siegel RL, Miller KD, Jemal A (2019) Cancer statistics, 2019. CA Cancer J Clin 69(1):7-34

9. Taylor JA, Kuchel GA (2009) Bladder cancer in the elderly: clinical outcomes, basic mechanisms, and future research direction. Nat ClinPractUrol 6(3):135-144

10. Joudi FN, Smith BJ, O'Donnell MA, Konety BR (2006) The impact of age on the response of patients with superficial bladder cancer to intravesical immunotherapy. J Urol 175(5):1634-1639 (discussion 1639-1640)

11. Oddens JR, Sylvester RJ, Brausi MA, Kirkels WJ, van de Beek C, van Andel G et al (2014) The effect of age on the efficacy of maintenance bacillus Calmette-Guérin relative to maintenance epirubicin in patients with stage $\mathrm{Ta} \mathrm{T} 1$ urothelial bladder cancer: results from EORTC genito-urinary group study 30911. EurUrol 66(4):694-701

12. Herr HW (2007) Age and outcome of superficial bladder cancer treated with bacilleCalmette-Guérin therapy. Urology 70(1):65-68

13. Heiner JG, Terris MK (2008) Effect of advanced age on the development of complications from intravesical bacillus Calmette-Guérin therapy. UrolOncol 26(2):137-140

14. Charlson ME, Pompei P, Ales KL, MacKenzie CR (1987) A new method of classifying prognostic comorbidity in longitudinal studies: development and validation. J Chronic Dis 40(5):373-383
15. Kang M, Jeong CW, Kwak C, Kim HH, Ku JH (2016) Preoperative neutrophil-lymphocyte ratio can significantly predict mortality outcomes in patients with non-muscle invasive bladder cancer undergoing transurethral resection of bladder tumor. Oncotarget 8(8):12891-12901

16. Yuk HD, Jeong CW, Kwak C, Kim HH, Ku JH (2018) Elevated neutrophil to lymphocyte ratio predicts poor prognosis in nonmuscle invasive bladder cancer patients: initial intravesical Bacillus Calmette-Guerin treatment after transurethral resection of bladder tumor setting. Front Oncol 8:642

17. Spencer BA, McBride RB, Hershman DL, Buono D, Herr HW, Benson MC et al (2013) Adjuvant intravesical Bacillus CalmetteGuérin therapy and survival among elderly patients with nonmuscle-invasive bladder cancer. J OncolPract 9(2):92-98

18. Lenis AT, Donin NM, Litwin MS, Saigal CS, Lai J, Hanley JM et al (2017) Association between number of endoscopic resections and utilization of Bacillus Calmette-Guerin therapy for patients with high-grade, non-muscle invasive bladder cancer. ClinGenitourin Cancer 15(1):e25-31

19. Patschan O, Holmäng S, Hosseini A, Liedberg F, Ljungberg B, Malmström P-U et al (2015) Use of bacillus Calmette-Guérin in stage $\mathrm{T} 1$ bladder cancer: long-term observation of a populationbased cohort. Scand J Urol 49(2):127-132

20. Sylvester RJ, Brausi MA, Kirkels WJ, Hoeltl W, Calais Da Silva F, Powell PH et al (2010) Long-term efficacy results of EORTC genito-urinary group randomized phase 3 study 30911 comparing intravesical instillations of epirubicin, bacillus Calmette-Guérin, and bacillus Calmette-Guérin plus isoniazid in patients with intermediate- and high-risk stage Ta T1 urothelial carcinoma of the bladder. EurUrol 57(5):766-773

21. Gontero P, Sylvester R, Pisano F, Joniau S, Vander Eeckt K, Serretta V et al (2015) Prognostic factors and risk groups in T1G3 non-muscle-invasive bladder cancer patients initially treated with Bacillus Calmette-Guérin: results of a retrospective multicenter study of 2451 patients. EurUrol 67(1):74-82

22. Naselli A, Hurle R, Paparella S, Buffi NM, Lughezzani G, Lista G et al (2018) Role of restaging transurethral resection for T1 nonmuscle invasive bladder cancer: a systematic review and metaanalysis. EurUrol Focus 4(4):558-567

23. Zapała P, Dybowski B, Poletajew S, Białek $Ł$, Niewczas A, Radziszewski P (2018) Clinical rationale and safety of restaging transurethral resection in indication-stratified patients with high-risk non-muscle-invasive bladder cancer. World J SurgOncol 16(1):6

24. Williams SB, Kamat AM, Chamie K, Froehner M, Wirth MP, Wiklund PN et al (2018) Systematic review of comorbidity and competing-risks assessments for bladder cancer patients. EurUro1Oncol 1(2):91-100

25. Søgaard M, Thomsen RW, Bossen KS, Sørensen HT, Nørgaard M (2013) The impact of comorbidity on cancer survival: a review. ClinEpidemiol 5(Suppl 1):3-29

Publisher's Note Springer Nature remains neutral with regard to jurisdictional claims in published maps and institutional affiliations. 